\title{
QUANTITATIVE TEMPERATURE IMAGING IN GAS-PHASE TURBULENT THERMAL CONVECTION BY LASER-INDUCED FLUORESCENCE OF ACETONE
}

\author{
Sean P. Kearney ${ }^{+}$and Felipe V. Reyes \\ Engineering Sciences Center \\ Sandia National Laboratories \\ PO Box 5800, MS 0834 \\ Albuquerque, NM 87185-0834 USA
}

Submitted to the Fifth World Conference on Experimental Heat Transfer, Fluid Mechanics, and Thermodynamics (ExHFT V), Thessaloniki, Greece, 24-28 September, 2001

Keywords: laser diagnostics, temperature imaging, laser-induced fluorescence, turbulent thermal convection

\begin{abstract}
In this paper, an acetone planar laser-induced fluorescence (PLIF) technique for nonintrusive, temperature imaging is demonstrated in gas-phase $(\mathrm{Pr}=0.72)$ turbulent Rayleigh-Bénard convection at Rayleigh number, $R a=1.3 \times 10^{5}$. The PLIF technique provides quantitative, spatially correlated temperature data without the flow intrusion or time lag associated with physical probes and without the significant path averaging that plagues most optical heat-transfer diagnostic tools, such as the Mach-Zehnder interferometer, thus making PLIF an attractive choice for quantitative thermal imaging in easily perturbed, complex three-dimensional flow fields. The "instantaneous" (20-ns integration time) thermal images presented have a spatial resolution of $176 \times 176 \times 500 \mu \mathrm{m}$ and a single-pulse temperature measurement precision of \pm 5.5 $\mathrm{K}$, or $5.4 \%$ of the total temperature difference. These images represent a 2-D slice through a complex, 3-D flow allowing for the thermal structure of the turbulence to be quantified. Statistics such as the horizontally averaged temperature profile, rms temperature fluctuation, two-point spatial correlations, and conditionally averaged plume structures are computed from an ensemble of 100 temperature images. The profiles of the mean temperature and rms temperature fluctuation are in good agreement with previously published data, and the results obtained from the two-point spatial correlations and conditionally averaged temperature fields show the importance of large-scale coherent structures in this turbulent flow.
\end{abstract}

\footnotetext{
+ Corresponding author. Phone: +1-505-844-6669, Fax: +1-505-844-8251, email: spkearn@sandia.gov. Sandia is a multiprogram laboratory operated by Sandia Corporation, a Lockheed-Martin Company, for the United States Department of Energy under Contract DE-AC04-94AL85000.
} 


\section{DISCLAIMER}

This report was prepared as an account of work sponsored by an agency of the United States Government. Neither the United States Government nor any agency thereof, nor any of their employees, make any warranty, express or implied, or assumes any legal liability or responsibility for the accuracy, completeness, or usefulness of any information, apparatus, product, or process disclosed, or represents that its use would not infringe privately owned rights. Reference herein to any specific commercial product, process, or service by trade name, trademark, manufacturer, or otherwise does not necessarily constitute or imply its endorsement, recommendation, or favoring by the United States Government or any agency thereof. The views and opinions of authors expressed herein do not necessarily state or reflect those of the United States Government or any agency thereof. 


\section{DISCLAIMER}

Portions of this document may be illegible in electronic image products. Images are produced from the best available original document. 


\section{INTRODUCTION}

DEC 2 ? 2000

Fundamental experimental studies of turbulent convective heat transfer are important in engineering applications such as fire safety and enhanced heat exchangers, as well as for scientific research in the areas of buoyancy generated turbulence and development of subgrid computational models for large-eddy simulation tools. Such detailed convection studies require quantitative, spatially and temporally resolved temperature and velocity measurements obtained in a nonintrusive manner due to the readily perturbed nature of thin boundary layers and buoyancy driven flows. Multi-component velocity diagnostics such as LDV and PIV have undergone considerable development over the last two decades and these systems are now commercially available and are standard equipment in many fluid mechanics and convection laboratories. However, nonintrusive temperature diagnostics for spatially/temporally resolved convection studies are generally not as well developed as velocity diagnostics have been.

Temperature diagnostics for convection studies have typically included intrusive devices such as cold wires, thermocouples and RTDs, which are limited to point measurements, can significantly perturb local velocity and temperature fields, and suffer from limited time response due to a finite probe thermal inertia. Nonintrusive, quantitative temperature imaging has been widely performed using interferometric methods [1], which yield line-of-sight integrated data and therefore provide a 2-D imaging measurement which is of limited utility in highly threedimensional turbulent flow fields.

For liquid-phase flows, planar laser-induced fluorescence (PLIF) and thermochroic liquid crystal thermography (LCT) are attractive options to line of sight techniques. PLIF and LCT allow for acquisition of "full field" quantitative temperature images on both an instantaneous and time-mean basis, without the extensive path averaging that plagues interferometric methods. Sakakibara et al. [2], [3] nonintrusively obtained 2-D images of $u, v$, and $T$ in turbulent pipe and stagnation flows of water using combined PIV and PLIF. They seeded their water flow with Rhodamine 590, a common laser dye, which fluoresces when illuminated with chopped lasersheet pulses from a CW $\mathrm{Ar}^{+}$laser. Using this Rhodamine PLIF method, "instantaneous" temperature images were obtained with an uncertainty of $\pm 1.2 \mathrm{~K}$ and these data were combined with the simultaneous PIV data to obtain in-plane maps of the Reynolds stress and turbulent heat flux. Sakakibara and Adrian [4] used a two-color Rhodamine PLIF technique in a turbulent thermal convection flow of water. The dynamics of the convection at $R a=1.3 \times 10^{7}$ were sufficiently slow to sweep the illuminating laser sheet across the flow field and obtain quasi- 
instantaneous three-dimensional temperature data, which revealed the full 3-D structure of complex, buoyant thermals at all stages of their development:

Günther and von Rohr [5] used the LCT technique to measure temperature and velocity simultaneously in a turbulent Rayleigh-Bénard flow of water at $R a=3.4 \times 10^{5}$. They seeded the water flow with $20-\mu \mathrm{m}$ thermochroic liquid crystals, which were also used as seed particles for simultaneous PIV, and illuminated the flow with a chopped white light sheet. The LCT technique is attractive for liquid flow applications because its high temperature sensitivity provides temperature resolution on the order of $0.1 \mathrm{~K}$, allowing for constant-property flows to be studied, and because the large liquid-crystal particles provide for the straightforward addition of simultaneous PIV. The LCT technique also does not require laser illumination, making the method potentially less costly than LIF methods.

While the Rhodamine PLIF and LCT methods mentioned here have proved very effective in liquid-phase flows, they typically cannot be applied in the gas-phase because dyes such as Rhodamine must be dissolved in a liquid solution and because the large size of the LCT particles hinders their entrainment in very low-momentum buoyant gas flows. For gas-phase PLIF measurements, acetone is an effective seed molecule due to its high vapor pressure, low toxicity, and a broadband UV absorption spectrum that is readily accessed by high-intensity Nd:YAG and excimer laser systems. Acetone PLIF has been widely used for gas-phase scalar mixing studies [6], [7]. Recently, Thurber et al. [8], [9], [10], [11] performed the first investigation of the potential of acetone PLIF as a temperature diagnostic. Thurber et al. quantified the temperature dependence of acetone fluorescence for excitation at several commercial pulsed-laser wavelengths [9], while also investigating the effects of pressure and oxygen concentration on the acetone PLIF signal [10]. Acetone PLIF thermometry was also demonstrated in a heated jet flow [9] and in a mixed convection flow over a circular cylinder [8] by Thurber and his co-workers as well.

In this paper, the acetone PLIF thermometry technique, put forth by Thurber et al. is applied to gas-phase, turbulent Rayleigh-Bénard convection. The acetone PLIF method allows for gasphase thermometry, and yields spatially correlated data without the flow intrusion or time lag associated with physical probes and without the significant path averaging that plagues most optical heat-transfer diagnostic tools. The paper begins with a summary of key acetone PLIF principles for temperature measurements, which is followed by the pertinent details of the present PLIF apparatus, and the procedures used for data acquisition, data reduction and 
temperature calibration. The results are then presented in terms of single-laser-pulse (or "singleshot") temperature maps and the appropriate statistics averaged over the ensemble of 100 singleshot temperature fields and over the horizontal dimension where appropriate. The paper closes with a concise an uncertainty analysis of the PLIF results.

\section{PLIF THERMOMETRY PRINCIPLES}

Acetone PLIF temperature measurements are performed by illuminating the flow field of interest with a pulsed UV laser sheet. The flow is seeded with the acetone molecule, which is electronically excited by the absorption of UV photons and re-emits a temperature dependent fluorescence signal in the visible spectrum (350 to $550 \mathrm{~nm}$ ) as a fraction of the excited population decays to the ground state via spontaneous emission. For "weak" (i.e. nonsaturating) laser excitation, the LIF signal is best modeled by an equation of the following form [8], [9],

$$
S_{f}=\eta_{o p t} \frac{\Omega}{4 \pi} \frac{\varepsilon}{h c / \lambda} V_{c} n_{a}\left(T, \chi_{a}\right) \sigma(\lambda, T) \phi(\lambda, T)
$$

where $\eta_{\text {opt }}$ is the efficiency of the signal collection optics, $\Omega / 4 \pi$ is the fractional solid angle of signal collection, $\varepsilon$ is the laser fluence $\left(\mathrm{J} / \mathrm{cm}^{2}\right), h c / \lambda$ is the energy per laser photon, $V_{c}$ is the signal collection volume (in this case a CCD pixel projected into the image plane and multiplied by the local laser-sheet thickness), $n_{a}$ is the local number density of acetone molecules, $\sigma$ is the acetone absorption cross section at the laser wavelength, $\lambda$, and $\phi$ is the fluorescence quantum efficiency, which is the fraction of absorbing molecules which emit fluorescence. In a given experiment, the collection optics, laser wavelength, and collection volumes are constant, and small ( $<3 \%$ ) pulse-to-pulse fluctuations in the laser output can be monitored. We also consider the case of constant pressure flow seeded with a constant, uniform mole fraction, $\chi_{a}$, of acetone ${ }^{\dagger}$ so that

$$
S_{f} \sim \frac{\sigma(T) \phi(T)}{T}
$$

\footnotetext{
${ }^{+}$The case of varying $\chi_{a}$ is treated by Thurber et al. [8], [9] and requires near-simultaneous excitation at two different laser wavelengths and ratioing of the two fluorescence signals.
} 
For the experiments reported in this paper, 248-nm laser excitation ( $\mathrm{KrF}$ excimer laser) is selected). The 248-nm wavelength is optimal for this constant $\chi_{a}$ application because it provides the best temperature sensitivity of any commercial pulsed UV-laser option, and because the temperature dependence of the absorption cross section, $\sigma$, is negligible, minimizing measurement uncertainties associated with correction for laser-beam absorption along the beam path.

\section{APPARATUS AND PROCEDURE}

A diagram of the PLIF optical system is provided in Fig. 1. Excitation is provided at $248-\mathrm{nm}$ by a pulsed, $\mathrm{KrF}$ excimer laser. This laser has a user-selectable, variable repetition rate from 1 to $200 \mathrm{~Hz}$ and delivers an unpolarized, $1-\mathrm{cm} \times 2.5-\mathrm{cm}$ rectangular laser beam with a maximum output of $450 \mathrm{~mJ}$ per 20-ns laser pulse. The laser is run at low electrode voltage and the wings of the beam profile are apertured so that a $30-\mathrm{mJ}$ beam is incident on the convection apparatus. The acetone LIF signal is proportional to laser excitation energy, however, the pulse energy must be limited to avoid saturation of the probed electronic transition in the acetone molecule. The laser beam propagates with its long side vertically oriented. The beam-expanding cylindrical telescope (CL1, CL2) decreases the horizontal beam divergence by a factor of two so that a tighter focus and a thinner laser sheet are obtained in the measurement region. The laser sheet is formed by expanding the beam vertically with an $f=-150 \mathrm{~mm}$, plano-concave cylindrical lens (CL3) and then focusing the laser beam with an $f=+500 \mathrm{~mm}$ plano-convex spherical lens (SL1). The distance between CL3 and SL1 is chosen so that the vertical sheet height is collimated. The laser sheet enters and exits a Rayleigh-Bénard convection cell (described below) through fused-silica windows. Copper plates, which form the hot and cold walls of the convection cell serve to vertically aperture the laser sheet on entrance to the cell so that the laser sheet fully fills the convecting fluid layer. This optical system provides a laser sheet that varies from 500 to 1000 $\mu \mathrm{m}$ thick across the CCD detector field of view, with the focus in the center of detector field of view.

LIF signal collection is provided by a scientific-grade, 16-bit, back-illuminated CCD camera. LIF signal collection is performed at $f 1.2$ using a Nikkor glass lens mounted to the CCD detector. The laser sheet thickness combines with the CCD pixel dimensions and the magnification of the collection lens to provide an out-of-plane spatial resolution of 500 to 1000 
$\mu \mathrm{m}$ and an in-plane resolution of $0.176 \times 0.176 \mu \mathrm{m}$. Use of a back-illuminated detector provides very high (70 to $85 \%$ ) quantum efficiency in the blue-green spectrum of the LIF signal, which allows for low-noise, unintensified signal detection. The glass collection lens and the BK-7 optical window at the viewing port of the convection cell effectively filter any scattered UV laser radiation from the convection cell walls while passing over $90 \%$ of the LIF signal within the $f / 1.2$ cone of acceptance. This wide spectral separation between the exciting UV laser pulse and the visible LIF signal is a key advantage of the LIF technique which facilitates measurements near walls, where other methods suffer from poor SNR due to high levels of laser-beam scattering.

The free convection flow was established inside a circular-plan cell with a diameter of 300 $\mathrm{mm}$ and a plate spacing of $29.5 \mathrm{~mm}$, providing a large aspect ratio of 10.2 , which assured horizontal isotropy of all statistics, and minimized the effects of the conducting aluminum side walls. The upper and lower walls are made from high-conductivity copper and are cooled and heated, respectively, by circulating heat-transfer fluid from independently controlled constanttemperature baths through milled slots in the interior of the plates. The entrance and exits for the heat-transfer fluid are placed in close proximity in order to minimize temperature gradients in the plates. Four T-type thermocouples were potted just under the surface of each plate using a highconductivity epoxy. Readings from these thermocouples indicated temperature differences of no more than $\pm 2 \mathrm{~K}$ during the experiments reported here.

The convection cell was seeded with acetone vapor by bubbling pure nitrogen through liquid acetone contained in a Pyrex flask. The flask was immersed in a constant-temperature bath of ethylene-glycol and water which was set at a temperature of $-5^{\circ} \mathrm{C}$. A saturated nitrogen/acetone mixture emerged from the liquid-containing flask and this mixture was diluted with dry air so that some oxygen was present in the cell. The presence of a small ( $>15$ Torr) fraction of oxygen is desirable because oxygen quenches phosphorescence from the excited acetone triplet state. This phosphorescence spectrally overlaps the fluorescence signal and will typically contaminate the results if is not rejected by oxygen quenching or by fast gating with an intensified detector*. The concentration of oxygen was kept sufficiently low to guarantee that the cell mixture was not flammable. The constituent mole fractions of the convection-cell gas mixture were calculated by

\footnotetext{
- Acetone phosphorescence occurs on a much longer time scale than the fluorescence signal. Fast (50-ns or less) gating using an intensified $\mathrm{CCD}$ can also be used to reject the phosphorescence signal without the need for introducing oxygen into the mixture. Both intensified and unintensified detection schemes were tried here and unintensified detection was chosen because it results in higher SNR.
} 
metering the nitrogen and dry-air flows and from the known acetone vapor pressure calculated from the correlation given by Ambrose et al. [12]. For the experiments reported in this paper, the fractions of $\mathrm{N}_{2}$, acetone, and $\mathrm{O}_{2}$ were 87.7, 5.5, and 6.8 mole percent, respectively. The uncertainty in the measured acetone mole fraction was estimated at $\pm 10 \%$ of the value due to inaccuracies in reading the rotameters used to monitor the nitrogen and air flows. The repeatability of the acetone mole fraction between different fillings of the convection cell was $\pm 5 \%$ of the value, as determined by gas chromatography.

Four sets of 100 images were acquired from the convection cell to obtain the temperature data reported in this paper. First, a "background" image was recorded with the laser sheet passed through the convection cell and no acetone present. This background image was averaged over 100 laser shots and the average image was subtracted from all acetone LIF images to correct for very low levels of visible fluorescence from the cell windows and from small dust particles that remained inside the convection cell, despite continuous purging with dry air when not in use. The $\mathrm{N}_{2} / \mathrm{O}_{2} /$ acetone mixture was then flowed through the convection cell while the cell was at room temperature $(293 \mathrm{~K}$ ) and a 100-laser-shot-averaged "reference" LIF image was acquired. This average reference image served two purposes: 1.) it allowed the LIF images taken in thermal convection to be referenced to the LIF signal at $293 \mathrm{~K}$ (after subsequent correction for laser-beam absorption as explained below), and 2.) it allowed for compensation of the LIF signal for nonuniformities in the laser-sheet profile. Following the acquisition of the room-temperature reference image, the cell was filled with $\mathrm{N}_{2} / \mathrm{O}_{2}$ /acetone mixture and sealed by closing the flowsupply valves. Flow of heat-transfer fluids to the heated and cooled plates was then commenced and two hours were allowed for the plates to come to temperature. This was then followed by an extra half-hour period to allow the convection flow to attain a statistically stationary state. Following this 2 1/2 hour wait, an ensemble of 100 images was acquired from the RayleighBénard flow. The laser repetition rate was set at $1 \mathrm{pulse} / \mathrm{sec}$ and the CCD detector was read at a rate that allowed the state of the flow at every other pulse to be acquired. In this manner, the closed cell mixture was exposed to less than 250 laser shots, which was sufficiently low to avoid changes in the LIF signal due to photodissociation of acetone. 


\section{DATA REDUCTION}

Using the "background", "reference", and "LIF signal" images and the power-law calibration curve described in the next section, each of the 100 single-shot acetone LIF images acquired were converted to a temperature field. Ratioed, single-shot LIF intensities were calculated from,

$$
S_{f}^{*}(i, j)=\frac{S_{f}(i, j)}{S_{f^{\prime}, 293 \mathrm{~K}}(i, j)}=\frac{L_{i, j}-\left\langle B_{i, j}\right\rangle}{\left\langle R_{i, j}\right\rangle-\left\langle B_{i, j}\right\rangle},
$$

where the brackets indicate ensemble averaging over a set of 100 images, $i$ and $j$ are the horizontal and vertical CCD pixel indices, respectively, $L_{i, j}$ is the single-pulse LIF signal, $B_{i, j}$ is the background image, and $R_{i, j}$ is the reference image obtained at $293 \mathrm{~K}$. The $S_{f}^{*}(i, j)$ were then used to calculate an initial estimate of the temperature field from a calibration curve given by Eq. 5 below, which is discussed in the next section. This initial temperature estimate was, however, not corrected for the effects of laser-beam absorption by along the beam's path through the cell. To correct for beam absorption effects a second iteration was required in which the $S_{f}^{*}(i, j)$ were corrected using Beer's law,

$$
S_{f}^{+}(i, j)=S_{f}^{*}(i, j) \exp \left(\sigma \chi_{a} \frac{P l}{k}\left\{\frac{1}{T}-\frac{1}{293 \mathrm{~K}}\right\}\right),
$$

where $\sigma=2.18 \times 10^{-20} \mathrm{~cm}^{2}$ is the absorption cross section measured by Thurber and co-workers [9], $T$ is the initial temperature estimate calculated using $S_{f}^{*}, k$ is Boltzmann's constant, $l$ horizontal pixel dimension projected into the object plane, and $P$ is the local atmospheric pressure $(83.7 \mathrm{kPa})$. The final temperature images were then low-pass filtered with a standard Gaussian kernel. The Gaussian filter attenuated off $40 \%$ of the fluctuation energy at the Nyquist frequency of $28.4 \mathrm{~cm}^{-1}$ and reduced high-spatial-frequency fluctuations caused by CCD detector shot noise.

\section{CALIBRATION}

A calibration curve plotting temperature as a function of the normalized LIF signal ratio, $S_{f} / S_{f, 293 \mathrm{~K}}$, was obtained by operating both copper plates of the convection cell at equal temperatures. The outer walls of the cell were insulated and a T-type thermocouple was used to 
monitor the temperature of the gas mixture inside the cell. During this process, the thermocouple readings from the copper plates and gas mixture were equal to within $\pm 1^{\circ} \mathrm{C}$ of each other and a radial traverse of the thermocouple through the cell showed negligible temperature changes in the gas mixture between the cell center and the cell wall regions. An unfocused 248-nm excimer beam was passed through the cell and 100 fluorescence images were recorded at gas temperatures between 283 and $360 \mathrm{~K}$ along with background images with no acetone present in the cell. Identical collection optics were used for the calibration and thermal convection experiments. The 100-shot-averaged image at $293 \mathrm{~K}$ was used as the "reference" for all the calibration data sets and Eqs. 3 and 4 were used along with shot-averaged LIF signals, $\left\langle L_{i, j}\right\rangle$, to construct the calibration plot shown in Fig. 2. A power-law was fit to the data and the resulting equation given by,

$$
T=290.34 S_{f}^{+-0.51991}
$$

was used to calculate temperatures from the LIF images obtained in Rayleigh-Bénard convection. The calibration data are plotted alongside the results of Thurber [11], who obtained calibration data for acetone/nitrogen mixtures on a much coarser temperature grid for temperatures between 293 and $973 \mathrm{~K}$. The results of the present study show increased sensitivity to temperature when compared to Thurber's data. These differences in the respective calibration curves may be due to individual biases resulting from the different types of collection optics and detectors used in the different experiments. However, these biases, in principle, should not have a large effect. The effects of lower static pressures (the experiments were conducted at $1600-\mathrm{m}$ altitude) and the presence of oxygen, both of which should lower the fluorescence yield, $\phi$, in Eqs. 1 and 2 could also have contributed to this result as well ${ }^{*}$. The extent to which each of these factors contributes to the modest differences between our study and the work of Thurber [11] are uncertain, but this level of disagreement is certainly reasonable.

\footnotetext{
+ Both decreased pressure and the presence of oxygen in the gas mixture will lower $\phi$. Decreased pressure results in a lower rate of vibrational relaxation in the excited acetone singlet state (from which the decay is by fluorescence) and a subsequently higher rate of intersystem crossing to the first excited triplet state. The excited triplet is a "sink" state from which the excited molecule decays by phosphorescence or collisional quenching instead of fluorescence, lowering the LIF signal. Presence of the oxygen molecule has also been observed to enhance crossing to the triplet as well with a similar drop in signal. The details of these processes are beyond the scope of this paper and the reader is directed to Thurber and Hanson [10] and the references therein for further explanation.
} 


\section{RESULTS AND DISCUSSION}

Single-Shot PLIF Images

Single-shot PLIF temperature images from turbulent Rayleigh-Bénard convection at $R a=1.3$ $\times 10^{5}$ are shown as color contour maps in Figs. 3 and 4. The two-second time delay between PLIF exposures is equal to about two eddy turnover times so that the images are not time correlated. At the gas-mixture Prandtl number of 0.72 , a fully turbulent flow is realized at $R a=$ $10^{4}$ [13], so that the flow in the present study is well into the turbulent regime. The CCD detector field of view spans the full channel height $(29.5 \mathrm{~mm})$ vertically and 2.94 channel heights $(86.7$ $\mathrm{mm}$ ) horizontally. The gravity vector points toward the bottom of the page. This field of view is significantly larger than those used by Thurber and co-workers [8], [9] in their initial demonstration of the technique. The images are shown to scale and the isotherms, shown by black lines, are in 5-K increments. The images represent a 2-D slice through a complex 3-D turbulent flow, with very little path averaging. These single-shot images are essentially instantaneous, with an averaging time of only $20-\mathrm{ns}$, or the duration of a single excimer-laser pulse.

At this Rayleigh number, the images shown in Figs. 3 and 4 exhibit coherent, plume-like structures with a very large vertical dimension. These structures penetrate nearly the full layer depth with the exception of thin, stable layers near both the hot and cold surfaces. These nearwall thermal layers appear almost black in appearance due to coalescence of the isotherms, which is indicative of a high boundary-layer temperature gradient. The thickness of this near wall-layer is represented by the inner-layer scales presented by Adrian and co-workers [14], [15], given by,

$$
z_{+}=\frac{\alpha}{w_{+}}=\frac{\alpha}{\left(\frac{\alpha^{2}}{\mu C_{p}} g \beta q^{\prime \prime}\right)^{1 / 4}}
$$

where $w_{+}$is an "inner" velocity scale. An inner layer length scale of order 1-mm was calculated using Eq. 6. This result is consistent with the observed scale of the near-wall regions at both walls in the single-shot images in Figs. 3 and 4 and in the conditionally averaged thermal plumes to be presented below. "Young" plume structures are seen to erupt from both near-wall layers. These young plumes have been observed in three dimensions in penetrative thermal convection 
by Adrian et al. [14], and by Sakakibara and Adrian [4], where they appear as elongated, 3-D "ridges". These ridge structures result from the unsteady growth of the stable inner fluid layer until a condition of local instability results in the release of a "mature" mushroom-type structure of the type shown in Figs. 3 and 4. The young structures observed in the acetone PLIF images presented here are likely a thin slice of a long, three-dimensional ridge structure which may or may not release a thermal in the plane of the laser sheet at a later time.

The horizontal dimension of these plume-like structures can also be very large. Two very large warm-fluid structures can be seen in Fig. 3a. The structure on the left side of the image has a horizontal dimension which occupies almost half the field of view, about 1.5 layer depths, and its true size is very likely larger, as it is not fully captured within the horizontal limits of the image. Visual observation of all 100 images in the ensemble shows that the organization of the thermal structure varies from small, alternating cool and warm plumes to very large, less organized regions of uniform temperature, which have a horizontal scale on the order of two to three layer depths. A comparison of these two cases is provided in Fig. 4. The image in Fig. 4a shows the more organized case of alternating plume structures which is indicative of the "roll" pattern seen in laminar convection. In contrast, the results selected for Figs. $4 \mathrm{~b}$ and $4 \mathrm{c}$, show extremely large regions of near-uniform-temperature cool (Fig. 4b) or warm (Fig. 4c) fluid. In this manner, comparison of Fig. $4 \mathrm{a}$ to Figs. $4 \mathrm{~b}$ and $4 \mathrm{c}$ suggests differing levels of the intensity of the local turbulent convection within the 2-D PLIF measurement region at different times during the turbulent convection process.

Observation of very large structures, such as those in Figs. $4 \mathrm{~b}$ and $4 \mathrm{c}$, in gas-phase turbulent convection is consistent with the classical results of Deardorff and Willis [16], whose horizontal temperature and velocity spectra, obtained from "rolling" hot- and cold-wire measurements in Rayleigh-Bénard convection of air, showed local energy maxima at length scales of several channel heights at $R a=6.3 \times 10^{5}$. Fitzjarrald [17] also used similar hot- and cold-wire methods in air to show that more than $90 \%$ of the energy content in the turbulent heat flux, $\left\langle w^{\prime} T^{\prime}\right\rangle$, fluctuations resides at length scales greater than the layer depth for $R a$ between $4 \times 10^{4}$ and $1.7 \times$ $10^{7}$.

\section{Mean and RMS Temperature Profiles}

Profiles of the mean temperature and rms temperature fluctuation obtained by horizontal averaging and subsequent averaging over the ensemble of 100 images, or a total of 511,000 
instantaneous temperature profiles, are shown in Fig. 5. The mean temperature profile shows boundary layers near the hot and cold walls, each with a temperature drop of one half the total temperature difference, as well as an isothermal core region at the average of the two wall temperatures. The shape of the mean profile conforms to accepted theory and numerous experimental investigations of Rayleigh-Bénard convection under the conditions of the Boussinesq approximation. This agreement is achieved in spite of the large (101 K) temperature difference employed in the present study, which resulted in a $36 \%$ change in the fluid density and variations in kinematic viscosity and thermal diffusivity of a factor of 1.7 across the layer depth. The mean heat flux was estimated at both walls from the slopes least-squares lines fit to the four data points in closest proximity to each wall. The choice of four data points allowed for a reasonable number of data points to be included in the fit, while keeping all of the fitted data within $0.5 \mathrm{~mm}$ of the wall, which was about half of the conduction layer scale of $1-\mathrm{mm}$ estimated from Eq. 6. The evaluated heat fluxes at the hot and cold walls were $1.01 \mathrm{~kW} / \mathrm{m}^{2}$ and 0.95 $\mathrm{kW} / \mathrm{m}^{2}$, respectively. This agreement of the estimated mean wall heat fluxes to within $6.1 \%$ is evidence of the consistency of the PLIF mean temperature measurements.

A vertical profile of the rms temperature fluctuation calculated from,

$$
\sigma_{\theta}(z)=\frac{1}{\Delta T} \sqrt{\left(\overline{(T(x, z)-\bar{T}(x))^{2}}\right\rangle},
$$

is provided in Fig. 5b. This shape of the rms profile is consistent with previously reported experimental data [16] and DNS results [18], [19] for turbulent convection at $P r=0.7$ and $R a=$ $6.3 \times 10^{5}$. The temperature fluctuation profiles are characterized by a local maxima in $\sigma_{\theta}$ near the outer edge of the thermal boundary layers. These local maxima in $\sigma_{\theta}$ result from buoyant production of turbulence due to the local instability mechanism mentioned above which leads to the random bursting of coherent plume structures from the stable layer near the wall. The temperature fluctuation then decays toward the middle of the fluid layer as the rising and falling thermal plumes transfer heat, primarily by turbulent mixing, which reduces the level of temperature variation in the core of the fluid layer. The maxima in the $\sigma_{T}$ results from the PLIF temperature measurements are bracketed by the experimental and computational results from the literature, while the location of the maxima in the present results are about 0.025 layer depths further from the wall. This displacement of the maxima is expected to occur since the Rayleigh number in the present study is about five times lower than for the literature data plotted in Fig. 
5b. The PLIF results also show an asymmetry in the $\sigma_{\theta}$ profiles, with larger temperature fluctuations near the hot wall. Larger temperature fluctuations near the hot wall may be expected because the factor of 1.7 increase in thermal diffusivity and the $36 \%$ decrease in density between the hot- and cold-wall temperatures results is a $17 \%$ increase in the inner scale for temperature fluctuations given by [14], [15],

$$
\theta_{+}=\frac{q^{\prime \prime}}{\rho C_{p} w_{+}}
$$

Closer to the wall the rms fluctuation profile obtained from the PLIF measurements become somewhat more suspect. The rms profile should decay rapidly from the local maxima at about $0.1 \mathrm{H}$ from each wall to a value of zero at the thermally massive copper plates, but this is not observed in the results. This error in the near-wall profile is a result of decreased signal-to-noise ratio, which results from decreased laser-beam intensity and decreased optical collection efficiency in the near-wall region as well as low levels of fluorescence from micron-sized particulate matter which had clung to the surface of the copper plates. However, this decrease in SNR is limited to a very thin region near the walls and the rms fluctuation results are valid through the majority of the fluid layer, as shown by the good agreement with previously published profiles in Fig. 5 b.

\section{Mean Properties of Coherent Structures}

The PLIF temperature profiles shown in Figs. 3 and 4 provide instantaneous views of the coherent thermal structures that occur in this turbulent flow field. The instantaneous views are useful for obtaining a sample of the variety of stochastically occurring structures. It is also useful to quantify the mean shape and size of these structures in terms of statistical quantities for purposes of comparison to other experimental and computational results. Such a characterization of the mean structural properties was obtained by computing two-point spatial correlation functions and by calculating conditionally averaged temperature fields.

Spatial correlation fields have been widely used for characterizing coherent structure in turbulent flow fields [20], [21], [22]. Two-point correlations were calculated from the PLIF data using, 


$$
R_{\theta \theta}\left(x, z ; z_{0}\right)=\left\langle\overline{\tilde{\theta}\left(x^{\prime}, z_{0}\right) \tilde{\theta}\left(x^{\prime}+x, z\right)}\right\rangle
$$

where the spatial averaging operation, indicated by the overbar, is performed over all reference points $\left(x^{\prime}, z_{0}\right)$ in the region of interest, and

$$
\tilde{\theta}(x, z)=\frac{T(x, z)-1 / 2\left(T_{h}-T_{c}\right)}{T_{h}-T_{c}}
$$

is a modified dimensionless temperature which is negative for temperatures below the mean fluid temperature and positive above it. The resulting correlations for each single-shot image are then ensemble-averaged, as indicated by the brackets in Eq. 9.

Spatial correlation functions computed for $z_{0} / H=0.25$, and 0.75 are shown in Fig. 6 . The yaxis scaling in the figures has been expanded by a factor of four for presentation purposes. As should be expected, the results show a strong, self-correlation peak at $(x, z)=\left(0, z_{0}\right)$. Symmetry of $R_{\theta \theta}$ about the z-axis, which results from the horizontal isotropy of the turbulence, has not been forced in the computations. As a check on the results, the correlation fields can be seen to be approaching symmetry, especially for $x / H$ between about \pm 1 . However this statistic has not yet fully converged near the walls where sampling error due to differing numbers of warm and cool plume events over the 100-image ensemble are manifested as opposite signs in the correlation on either side of the z-axis.

The distance from the self-correlation peak to the zero contour is a measure of the length scale of the temperature fluctuations. In both of the correlation fields presented, the data are positively correlated throughout much of the domain, indicating that the vertical and horizontal length of the coherent thermal structures is of the order of the layer depth. The horizontal length scale of the thermal structures, as defined by the correlation fields, is larger than the \pm 2.4 -layerdepth size of the calculation domain for $R_{\theta \theta}$. This observation is consistent with the appearance of very large regions of uniform temperature which occur during periods of "vigorous" convection, as shown in Figs $4 \mathrm{~b}$ and $4 \mathrm{c}$.

While the spatial correlation functions presented above are a good way of quantifying the average size of coherent structures in a turbulent flowfield, they do not strictly differentiate between warm, light plumes, or 'updraughts', and heavier, cooler plumes or 'downdraughts' for the case of thermal convection. The correlation functions can also be difficult to intuitively 
interpret as well. With these weaknesses in mind, conditional averages were also computed to provide added information regarding any potential differences in the coherent structure of updraught and downdraught events, and to provide a more intuitive picture about the structure and size of an "average" plume. Conditional averages were computed by specifying an event condition, setting the location of the event to $x=0$, and then averaging only those temperature fields that satisfied the event condition. We computed two conditionally averaged plume structures by specifying the following conditions on the maximum and minimum temperatures at two vertical positions,

$$
\begin{aligned}
& \theta_{\max }\left(\frac{z}{H}=0.25\right)>0.6 \quad \text { "warm plume" event } \\
& \theta_{\max }\left(\frac{z}{H}=0.75\right)<0.4 \quad \text { "cool plume" event, }
\end{aligned}
$$

and averaging over a \pm 1.3 layer depth window in the horizontal dimension on either side of the event, excluding any locations which were outside the field of view. Only one event per singleshot PLIF image was accepted to guarantee that the events incorporated into the average were statistically independent.

The resulting conditionally averaged temperature fields are shown in Fig. 7. The results show that, in the mean, both hot and cold plume structures nearly reach the opposing heat-transfer surface following their release. This conclusion is consistent with the spatial correlation fields presented above. The conditional averages also show a thin conduction layer adjacent to each wall from which both the hot and cold thermal structures burst upon local instability of this layer. The mean thickness of these near-wall layers is about $2 \mathrm{~mm}$ or 0.07 fluid-layer depths, based on observation of the averages presented in Fig. 7. The horizontal dimension of the average plume structures inferred from the conditional averages is also of the order of the fluid layer depth as well. In Fig. 7, the color scale has been selected so that regions at a temperature less than the mean of the wall temperatures are shown in violet and blue while warmer regions are displayed in green, yellow and red. In the average warm plume shown in Fig. 7a, descending cool (light blue) fluid can be seen on either side of the rising warm (green) plume, suggesting that the horizontal scale of a rising plume is about 2 layer depths inside the layer core. Similarly, rising warm fluid can be seen lateral to the average cold plume shown in Fig. $7 \mathrm{~b}$ as well. This 
alternating warm/cool structure observed in the conditionally averaged temperature fields strongly suggests that the underlying structure of this turbulent convection field is a "roll pattern" of similar type to the structure observed in laminar Rayleigh-Bénard convection (see for example Fig. 6 of ref. [23]).

\section{PLIF Measurement Uncertainty}

The uncertainty in the PLIF temperature measurements presented above is quantified in this section in terms of precision (random) and bias (systematic) contributions. The major contributions to uncertainty in the PLIF temperature measurement arise from random errors due to detector shot noise and pulse-to-pulse fluctuations in the $\mathrm{KrF}$ excimer-laser output, and from systematic errors due to uncertainty in the acetone mole fraction and the calibration curve fit. The contributions to the total uncertainty from each of these error sources are summarized in Table 1 and the procedures used in obtaining these estimates are briefly summarized below. The uncertainty estimates presented in Table 1 are evaluated at $330 \mathrm{~K}$, which is the mean of the hotand cold-wall temperatures.

Three of the four error sources cited above (the exception being the calibration curve fit) directly impact the measured fluorescence signal and therefore the temperature, through the calibration in Eq. 5. Temperature uncertainties associated with each of these error sources were computed using,

$$
\delta_{T}^{(k)}=\frac{d T}{d S_{f}^{+}} \delta_{S_{f}^{+}}^{(k)}
$$

where the sensitivity $d T / d S_{f}^{+}$is obtained by differentiation of Eq. 5, and $\delta_{T}^{(k)}$ and $\delta_{S_{f}^{+}}^{(k)}$ are the uncertainties in temperature and fluorescence signal due to the $k$ th error source. The process then reduces to estimating the $\delta_{S_{f}^{+}}^{(k)}$ and applying Eq. 12.

The dominant sources of random error are shot noise and pulse-to-pulse fluctuations in the excimer laser output. Photon shot noise results from the statistical nature of the emission of photoelectrons from a CCD pixel's potential well. The uncertainty in signal level associated with shot noise is given by [24], 


$$
\delta_{S_{f}^{+}}^{\text {shot noise }}=\frac{1}{\sqrt{\eta_{Q} S_{f}}}
$$

where $\eta_{Q}$ is the CCD detector quantum efficiency in photoelectrons per incident signal photon. The observed signal levels at $330 \mathrm{~K}$ were used to calculate representative values of $S_{f}$ for the shot noise estimate given in Eq. 13. The uncertainty contribution due to pulse-to-pulse fluctuations in the excimer laser power output was estimated by noting that $S_{f}$ is linearly proportional to the

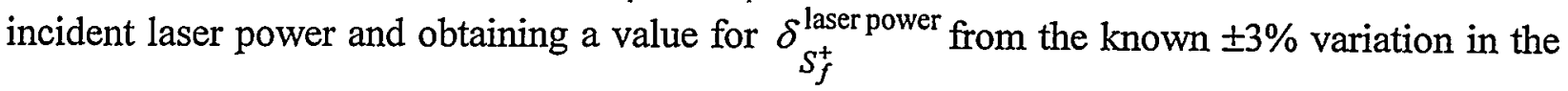
excimer-laser output.

The dominant sources of bias are the uncertainty in the value of the acetone mole fraction and the uncertainty incurred from calibration and curve fitting. The contribution from the $\pm 10 \%$ uncertainty in the gas-mixture acetone mole fraction arises through the Beer's law absorption correction in Eq. 4. An estimate of $\delta_{S_{f}^{+}}^{\chi_{a}}$ was obtained by calculating the correction term in Eq. 4 at the measured value of $\chi_{a}=0.055$ and at $\chi_{a}=0.055 \pm 10 \%$ at various path lengths along the laser beam. The bias contribution from the calibration curve fit was estimated from the $\pm 2 \mathrm{~K} \mathrm{rms}$ difference between the thermocouple temperatures and the power-law curve fit (Eq. 5) shown in Fig. 2.

Composite bias and precision limits were calculated by vector summing the individual bias and precision contributions given in Table 1. This process results in an overall measurement precision of $\pm 5.5 \mathrm{~K}$ and a composite bias limit of $\pm 2.6 \mathrm{~K}$ for the single-shot PLIF data presented in this paper. It should be noted that random error caused by laser power fluctuations causes a shot-to-shot-fluctuating bias toward higher or lower temperatures over the entire field of view, but does not cause high frequency noise in the images. In this respect, the power fluctuations could also be considered a single-shot bias, as opposed to a precision error. This effect is illustrated in the single-shot temperature images presented in Figs. 3 and 4, where temperature differences on the order of the composite precision error are well resolved. The impact of laserpower fluctuations can be significantly reduced by placing a room-temperature reference cell in the optical system and monitoring the PLIF signal at $293 \mathrm{~K}$ on a single-shot basis, as opposed to using the shot-averaged reference signal, $\left\langle R_{i, j}\right\rangle$, as was done here. Use of this reference cell will also permit pulse-to-pulse monitoring of small variations in the laser-sheet profile. The shot- 
noise contribution can also be reduced by increasing the overall signal level by seeding with a higher acetone mole fraction and by exciting with higher excimer laser energies. Maximum values for $\chi_{a}$ and laser power are, however, limited to prevent condensation of acetone in the system and to avoid saturating the probed acetone electronic transition.

\section{SUMMARY AND FUTURE WORK}

An ensemble of 100 acetone PLIF temperature images has been obtained in gas-phase, turbulent Rayleigh-Bénard convection at $R a=1.3 \times 10^{5}$ and $\operatorname{Pr}=0.72$. The precision and bias limits for the temperature data reported in this paper are $\pm 5.5 \mathrm{~K}$ and $\pm 2.6 \mathrm{~K}$, respectively. The single-shot PLIF temperature images clearly show the spatially resolved thermal structure of the complex turbulent flow field without the path-averaging effects that plague more traditional interferometric measurements. PLIF measurements of the profiles of mean temperature and rms temperature fluctuation are in good agreement with theory and with previous experiments and numerical simulations. Two-point spatial correlations of temperature show the importance of large-scale thermal structures in the Rayleigh-Bénard flow and conditionally averaged temperature fields strongly suggest that the mean structure is similar to the roll patterns observed in laminar Rayleigh-Bénard convection.

Current efforts in this arena include addition of a room-temperature acetone reference cell and the inclusion of simultaneous PIV. The PLIF measurement precision can be improved by adding a room-temperature reference cell to the optical system and monitoring a 293-K PLIF image on a single-shot basis. Monitoring of this reference signal will allow for improved compensation for pulse-to-pulse fluctuations in excimer-laser power and beam profile. The addition of simultaneous PIV will allow for a complete, spatially resolved and time-correlated description of the Rayleigh-Bénard and other complex, buoyancy driven turbulent flows, including turbulent heat flux, Reynolds stresses, and energy budgets, in addition to a quantitative description of the large-scale coherent structures in these complex flows. 


\section{REFERENCES}

1. Goldstein, R.J., "Optical Systems for Flow Measurement: Shadowgraph, Schlieren, and Interferometric Techniques", in Fluid Mechanics Measurements, R.J. Goldstein, Editor Hemisphere: New York, 1983.

2. Sakakibara, J., K. Hishida, and M. Maeda, "Measurements of Thermally Stratified Pipe Flow Using Image Processing Techniques", Exp. Fluids, 16, 82-96, 1993.

3. Sakakibara, J., K. Hishida, and M. Maeda, "Vortex Structure and Heat Transfer in the Stagnation Region of an Impinging Plane Jet (Simultaneous Measurements of Velocity and Temperature Fields by Digital Particle-Image Velocimetry and Laser-Induced Fluorescence)", Int. J. Heat Mass Transfer, 40(13), 3163-3176, 1997.

4. Sakakibara, J. and R.J. Adrian, "Whole Field Measurement of Temperature in Water Using Two-Color Laser-Induced Fluorescence", Exp. Fluids, 26, 7-15, 1999.

5. Günther, A. and B. von Rohr. Temperature Measurements in Rayleigh-Bénard Convection using an LCT Technique. Proceedings of the 2000 National Heat Transfer Conference, Pittsburgh, PA, 2000.

6. Lozano, A., B. Yip, and R.K. Hanson, "Acetone: A Tracer for Concentration Measurements in Gaseous Flows by Planar Laser-Induced Fluorescence", Exp. Fluids, 13, 369-376, 1992.

7. Meyer, T.R., J.C. Dutton, and R.P. Lucht, "Vortex Interaction and Mixing in a Driven Gaseous Axisymmetric Jet", Phys. Fluids, 11(11), 3401-3415, 1999.

8. Thurber, M.C., F. Grisch, and R.K. Hanson, "Temperature Imaging with Single- and Dual-Wavelength Acetone Planar Laser-Induced Fluorescence", Opt. Lett., 22(4), 251, 1997.

9. Thurber, M.C., et al., "Measurements and Modeling of Acetone Laser-Induced Fluorescence with Implications. for Temperature-Imaging Diagnostics", Appl. Opt., 37(21), 4963-4978, 1998.

10. Thurber, M.C. and R.K. Hanson, "Pressure and Composition Dependences of Acetone Laser-Induced Fluorescence with Excitation at 248, 266, and 308 nm", Appl. Phys. B, 69, 229-240, 1999.

11. Thurber, M.C., "Acetone Laser-Induced Fluorescence for Temperature and Multiparameter Imaging in Gaseous Flows", Ph.D. Dissertation, Thermophysics Division, Department of Mechanical Engineering, Stanford University, Stanford, CA, 1999.

12. Ambrose, D., C.H.S. Sprake, and R. Townsend, "Thermodynamic Properties of Organic Oxygen Compounds XXIII. The Vapour Pressure of Acetone", J. Chem. Thermodynamics, 6, 693-700, 1974. 
13. Krishnamurti, R., "On the Transition to Turbulent Convection. Part 2. The Transition to Time-Dependent Flow", J. Fluid Mech., 42(2), 309-320, 1970.

14. Adrian, R.J., R.T.D.S. Ferreira, and T. Boberg, "Turbulent Thermal Convection in Wide Horizontal Fluid Layers", Exp. Fluids, 4, 121-141, 1986.

15. Chung, M.K., H.C. Yun, and R.J. Adrian, "Scale Analysis and Wall-Layer Model for the Temperature Profile in Turbulent Thermal Convection", Int. J. Heat Mass Transfer, 35(1), 43-51, 1992.

16. Deardorff, J.W. and G.E. Willis, "Investigation of Turbulent Thermal Convection Between Horizontal Plates", J. Fluid Mech., 28(4), 675-704, 1967.

17. Fitzjarrald, D.E., "An Experimental Study of Turbulent Convection in Air", J. Fluid Mech., 73(4), 693-719, 1976.

18. Christie, S.L. and J.A. Domaradzki, "Scale Dependence of the Statistical Character of Turbulent Fluctuations in Thermal Convection", Phys. Fluids, 6(5), 1848-1855, 1994.

19. Kimmel, S.J. and J.A. Domaradzki, "Large Eddy Simulations of Rayleigh-Bénard Convection Using a Subgrid Scale Estimation Model", Phys. Fluids, 12(1), 169-184, 2000.

20. Hinze, J.O., Turbulence. 2nd ed, McGraw-Hill, New York, 1975.

21. Schmidt, H. and U. Schumann, "Coherent Structure of the Convective Boundary Layer Derived from Large-Eddy Simulations", J. Fluid Mech., 200, 511-562, 1989.

22. Prasad, A.K. and P.V. Gonuguntia, "Turbulence Measurements in Nonpenetrative Thermal Convection", Phys. Fluids, 8(9), 2460-2470, 1996.

23. Chu, T.Y. and R.J. Goldstein, "Turbulent Convection in a Horizontal Layer of Water", $J$. Fluid Mech., 60(1), 141-159, 1973.

24. Eckbreth, A.C., Laser Diagnostics for Combustion Temperature and Species, Abacus Press, Cambridge, MA, 1988. 


\section{NOMENCLATURE (symbols not defined here are done so in the text)}

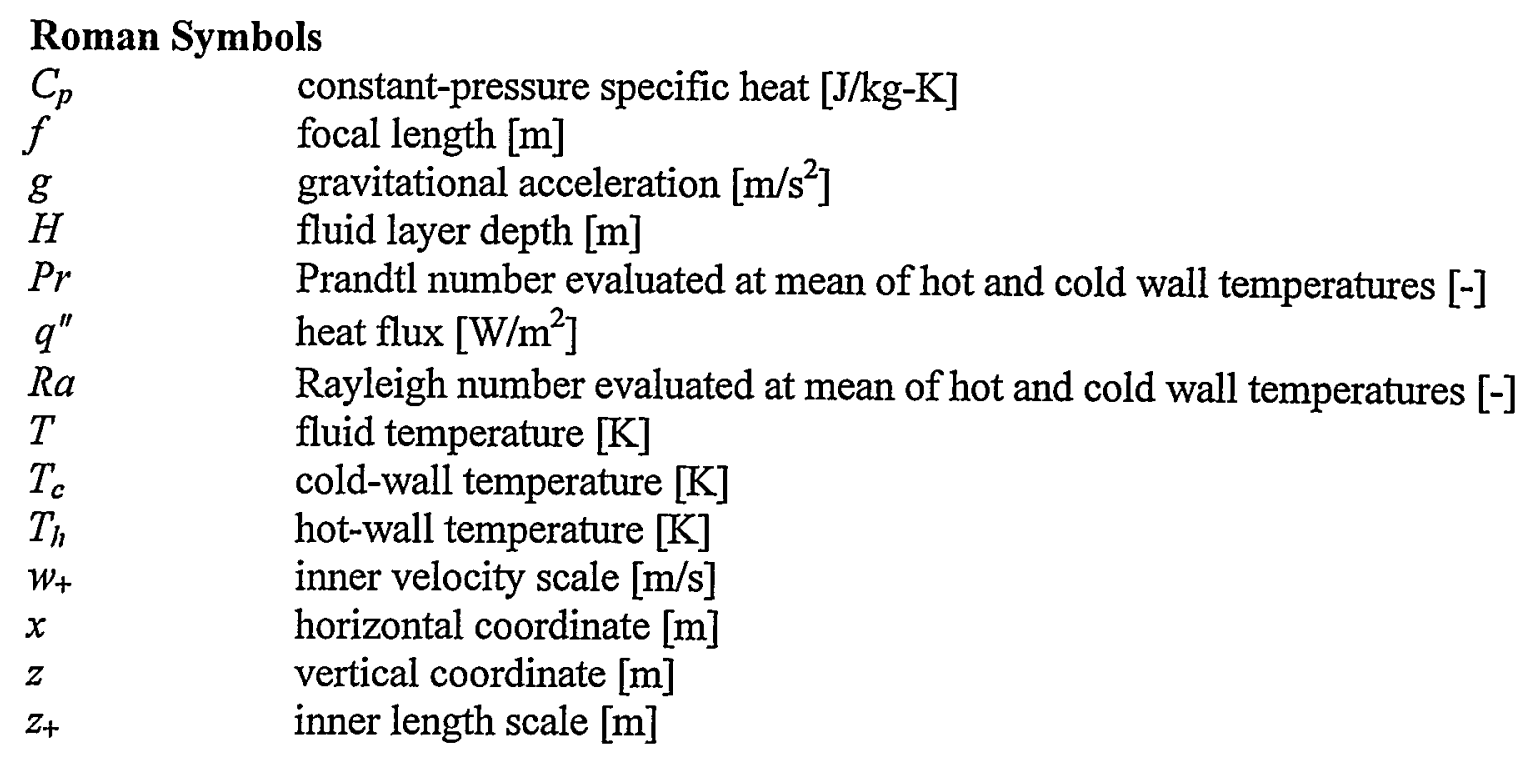

\section{Greek symbols}

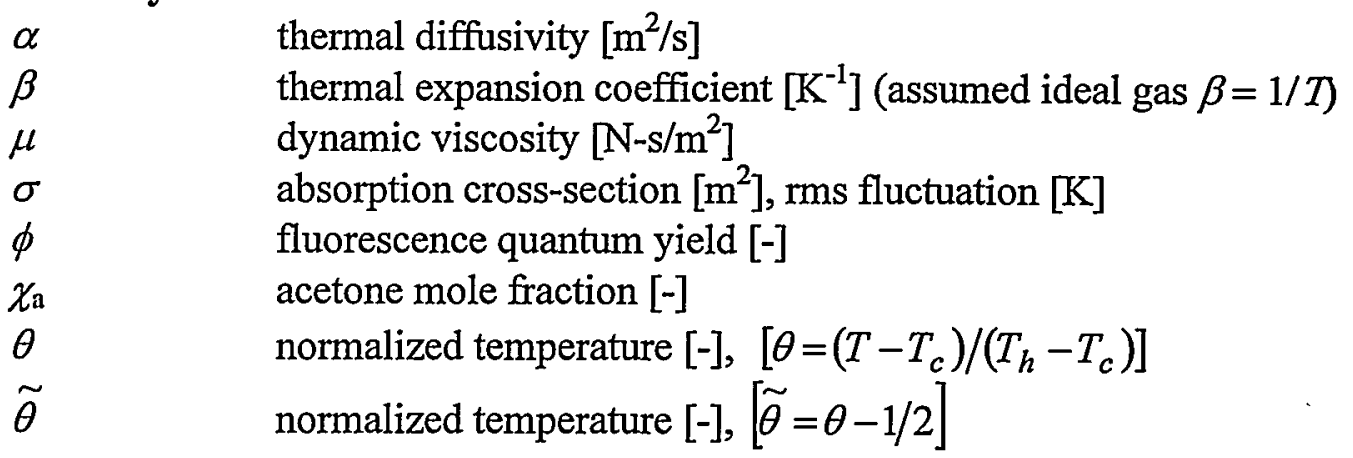

\section{Averaging Operations}

$\langle s\rangle \quad$ denotes averaging of quantity $s$ over 100-image ensemble

$s \quad$ denotes averaging of single-shot quantity $s$ over the horizontal dimension 
Table 1 - Uncertainty estimates at $300 \mathrm{~K}$.

\begin{tabular}{|c|c|c|c|}
\hline \hline Error Source & Error Type & $\delta_{S_{f}^{[}}[\%]$ & $\delta_{T}[\mathrm{~K}]$ \\
\hline Shot noise & Precision & 2.9 & 2.5 \\
\hline Laser power & Precision & 3.0 & 4.9 \\
\hline Calibration & Bias & - & 2 \\
\hline Acetone mole fraction & Bias & 1.0 & 1.7 \\
\hline
\end{tabular}




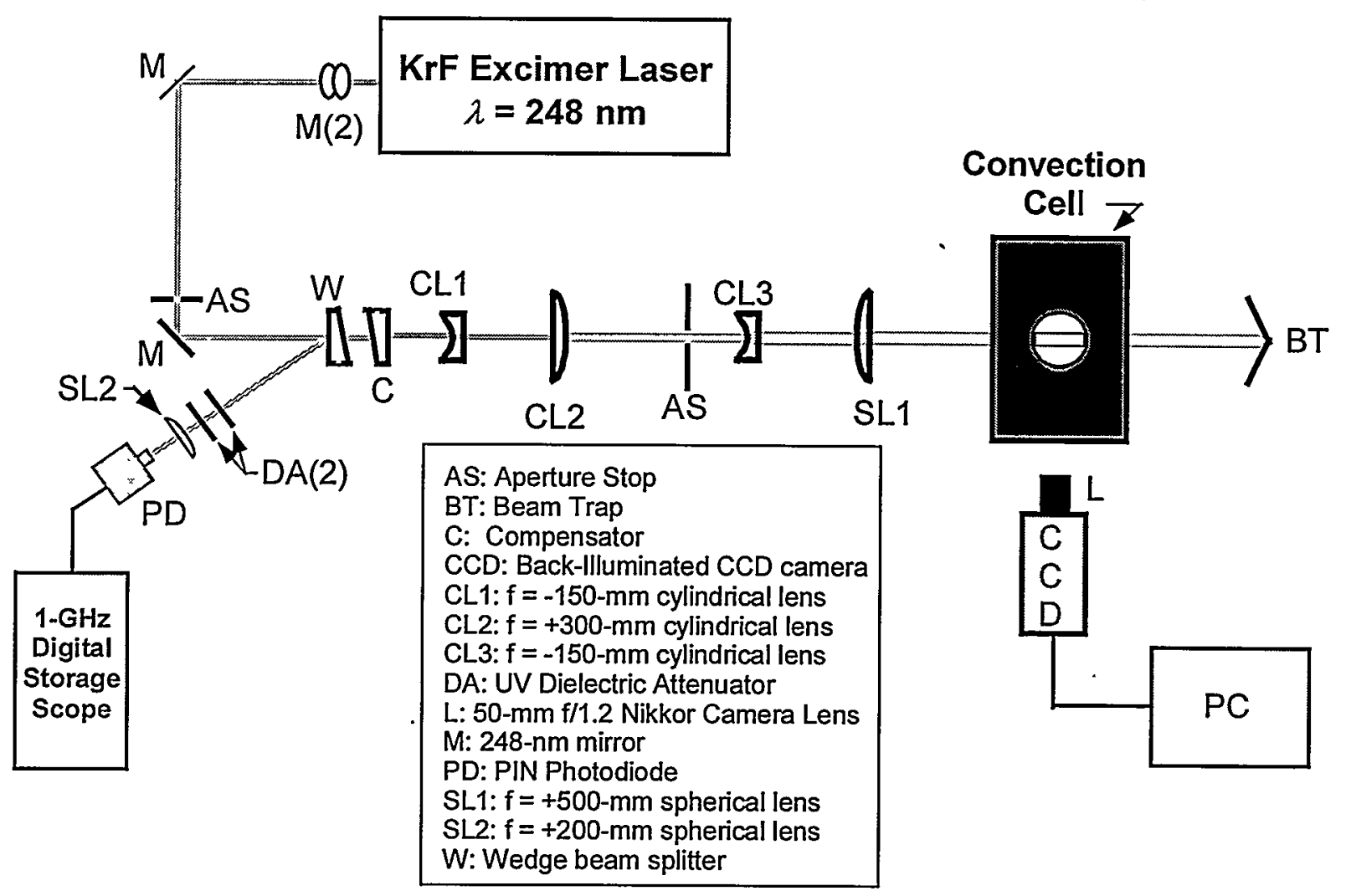

Figure 1 - Diagram of the optical system used for acetone PLIF temperature measurements in a circular-plan Rayleigh-Bénard convection cell. 


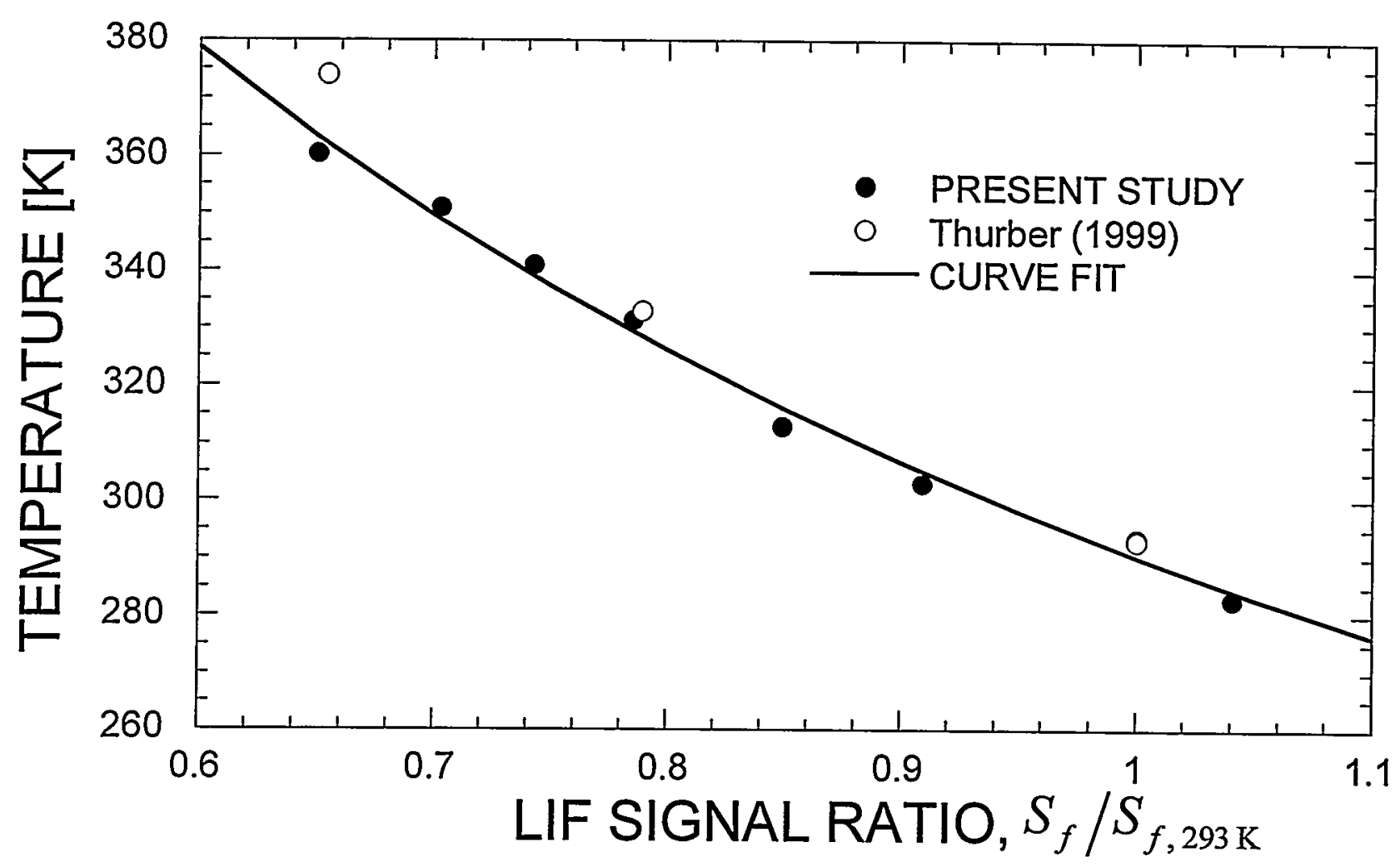

Figure 2 - Acetone LIF temperature calibration curve used to determine the reported temperature fields. $S_{f} / S_{f, 293 \mathrm{~K}}$ 

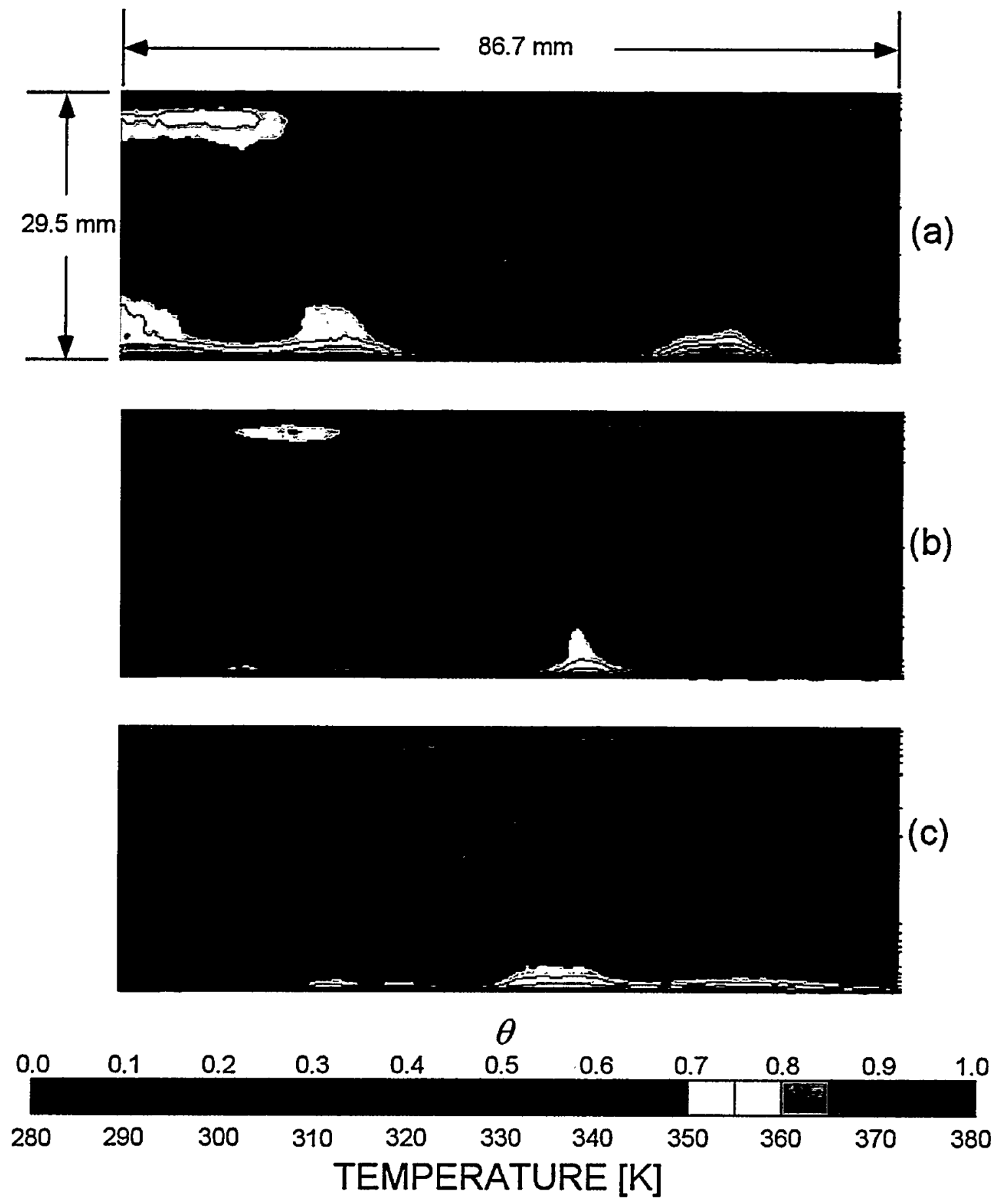

Figure 3 - Single-shot temperature fields from a gas-phase, turbulent Rayleigh-Bénard flow at $R a=1.3 \times 10^{5}$. The hot (lower) wall is at a temperature of $380 \mathrm{~K}$ and the cold (upper) wall at 279 $\mathrm{K}$. The images are shown to scale and the isotherm contours shown by black lines are in increments of $5 \mathrm{~K}$. Both rising, hot plumes and falling, cold plume structures can be seen at different stages of their development. The images represent a 2-D slice through a complex 3-D turbulent flow, with an out-of-plane spatial resolution conservatively estimated at $500 \mu \mathrm{m}$ in the center of the images and $1000 \mu \mathrm{m}$ near the edges. These single-shot images are essentially instantaneous, with an averaging time of only 20 -ns, or the duration of a single excimer laser pulse. 


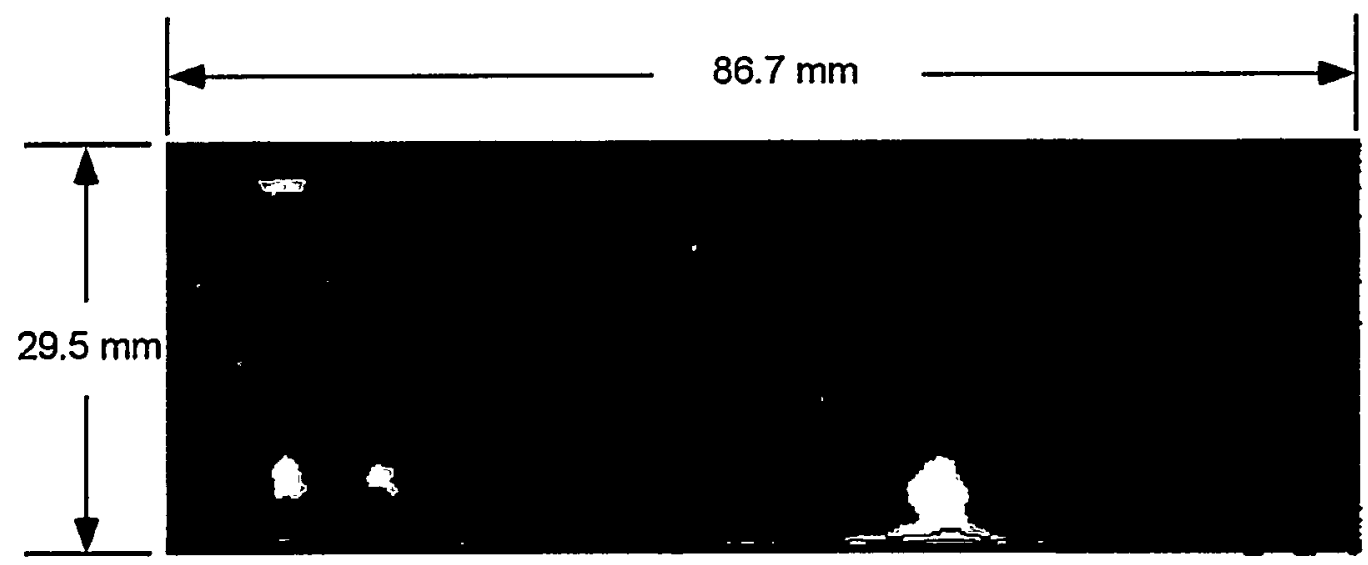

(a)

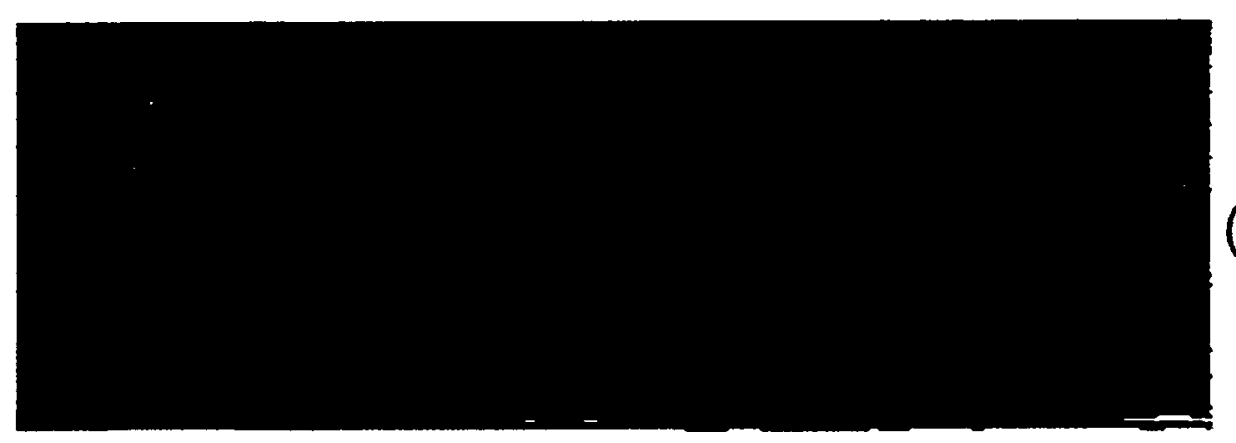

(b)

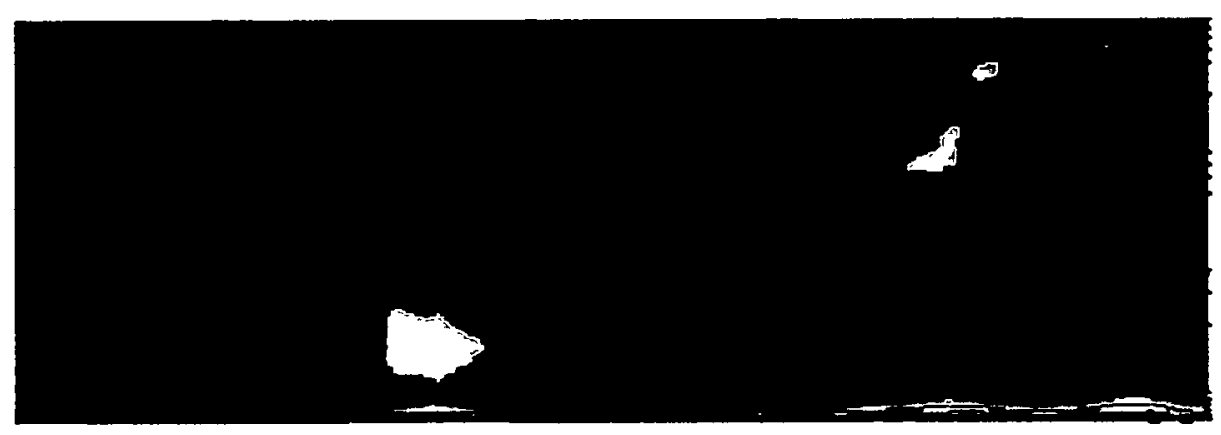

(c)

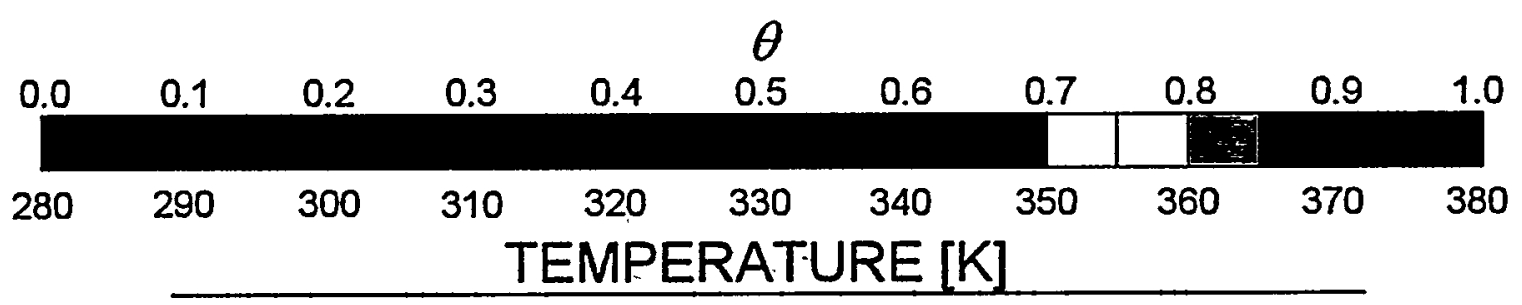

Figure 4 - Single shot temperature fields from a turbulent Rayleigh-Bénard flow of air at $R a=$ $1.3 \times 10^{5}$ as in Fig. 3b. Images (b) and (c) show regions of cold and warm fluid of very large extent. 

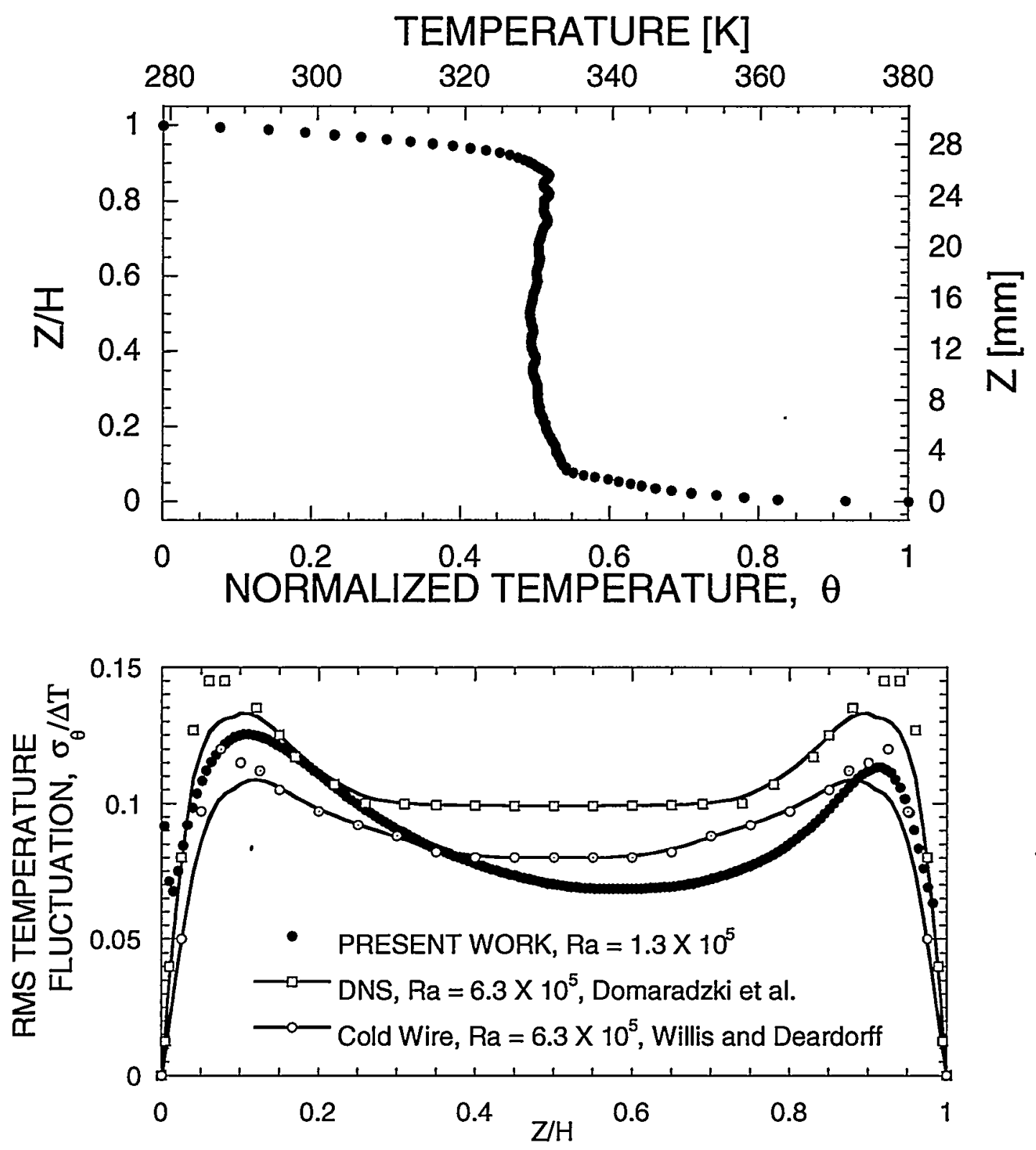

(b)

Figure 5 - Mean temperature profile from a turbulent Rayleigh-Bénard flow of air at $\mathrm{Ra}=1.3 \times$ $10^{5}$ obtained by averaging over the horizontal dimension and over an ensemble of 100 PLIF images. 


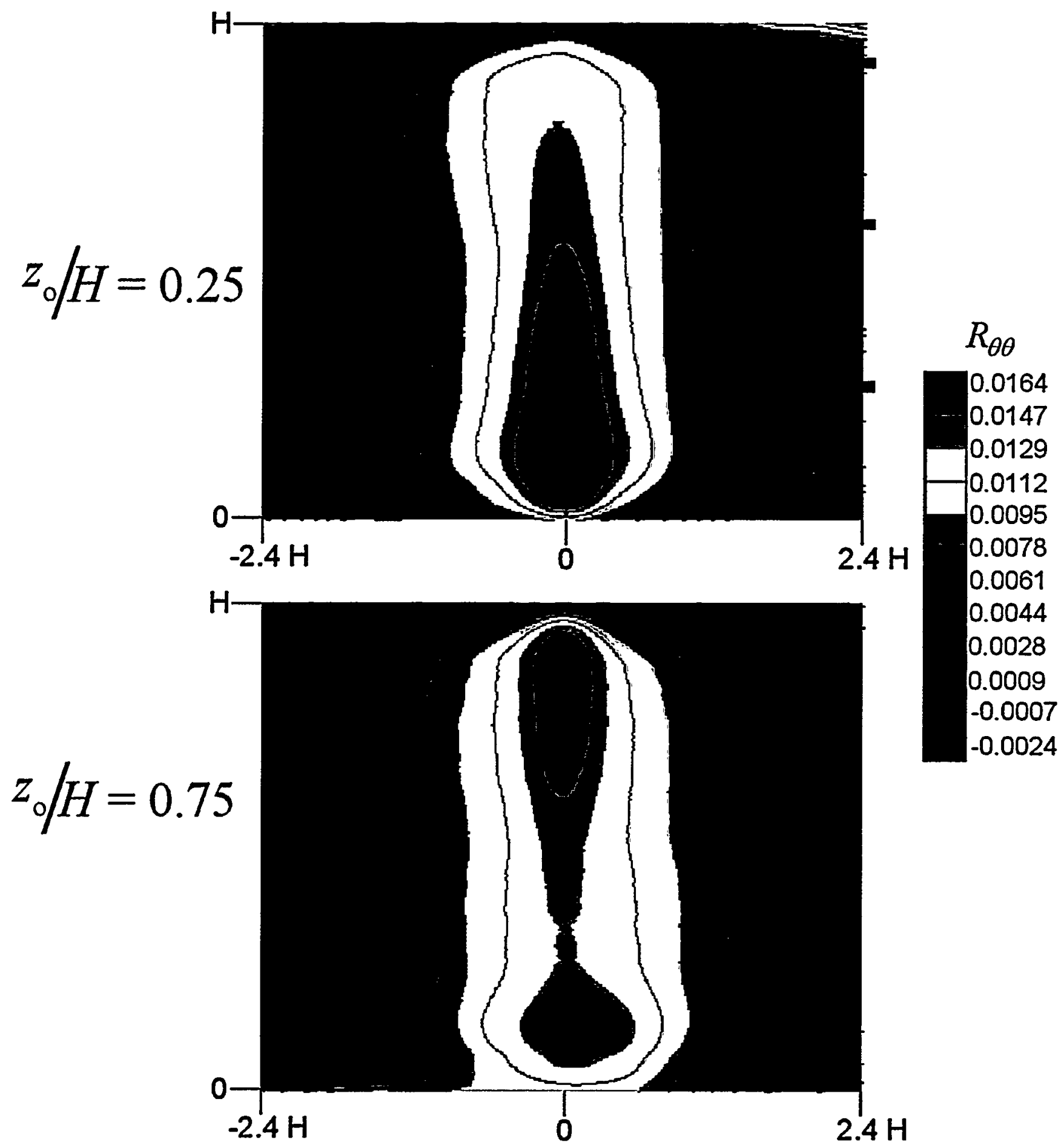

Figure 6-Two-point spatial correlation functions calculated for $z_{0} / H=0.25$, and 0.75 . 


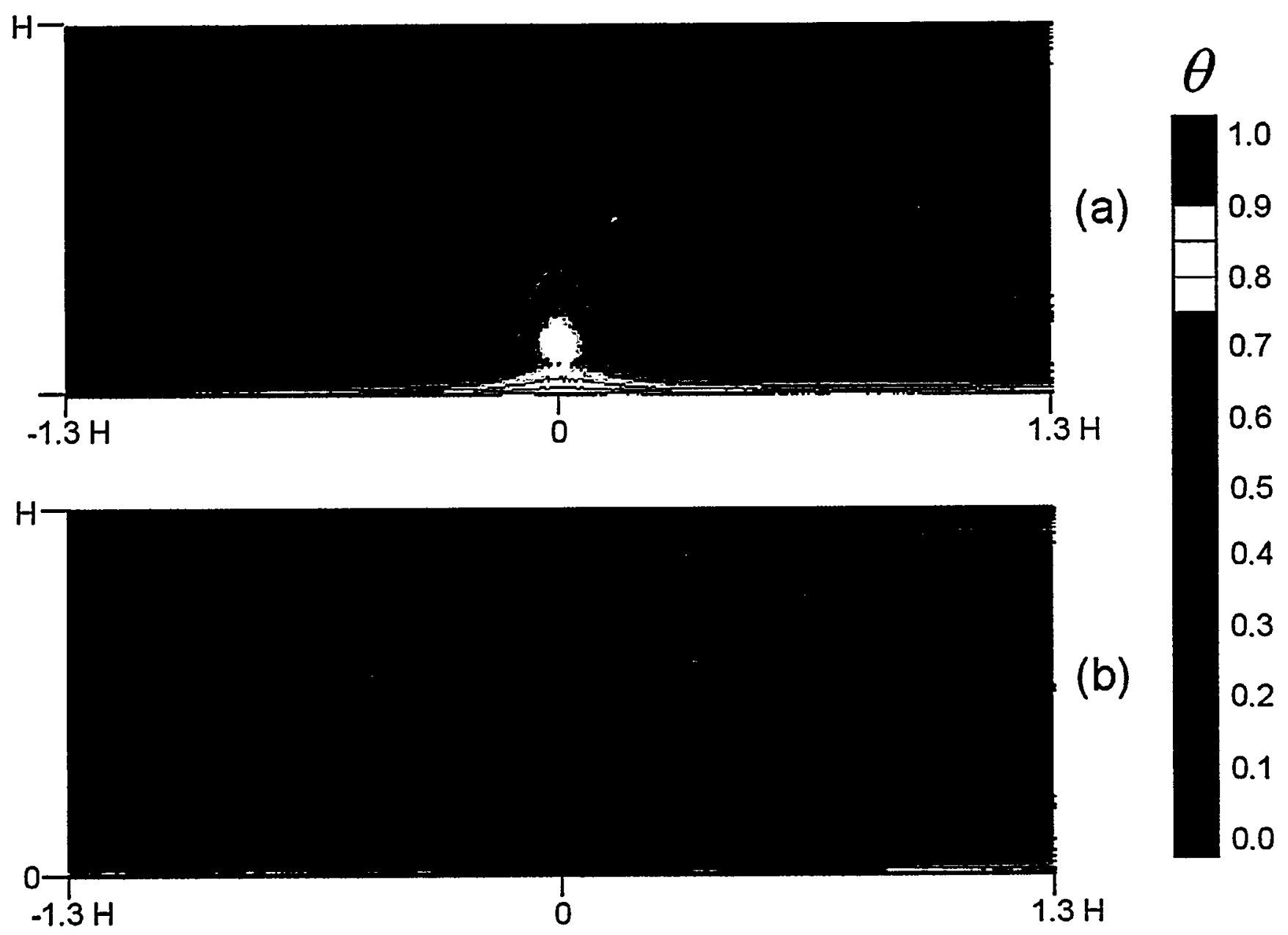

Figure 7 - Conditionally averaged updraught (a) and downdraught (b). The indicator for an updarught event is a local maximum of $\theta>0.6$ at $z / H=0.25$. The indicator for a downdraught event is a local minimum of $\theta<0.4$ at $z / H=0.75$. 